\title{
Owl's Eye Sign in a Reversible Etiology of Spastic Quadriparesis
}

\author{
Sumeet R. Dhawan ${ }^{1} \quad$ Lokesh Saini $^{1} \quad$ Savita Verma Attri ${ }^{1} \quad$ Ajay Kumar ${ }^{2}$ Naveen Sankhyan ${ }^{1}$
}

\author{
1 Department of Pediatrics, Postgraduate Institute of Medical \\ Education and Research, Chandigarh, India \\ 2 Department of Radiodiagnosis, Postgraduate Institute of Medical \\ Education and Research, Chandigarh, India
}

Neuropediatrics 2019;50:135-136.

A 10-year-old boy presented with progressive difficulty in walking for 1 year with preserved cognition. On examination, his head circumference was $51 \mathrm{~cm}$. He had an expressionless face, spastic speech, spastic gait, and impaired finger tapping. Other systemic examination was unremarkable. KayserFleischer rings were absent. An magnetic resonance imaging (MRI) of brain showed brainstem and spinal cord involvement ( - Fig. 1). Evaluation for antinuclear antibodies, serum copper and ceruloplasmin, ammonia, lactate, aquaporin- 4 antibody, tandem mass spectrometry, and urine for organic acids were
Address for correspondence Dr. Lokesh Saini, DM, Assistant Professor, Department of Pediatrics, Postgraduate Institute of Medical Education and Research, Chandigarh, India-160012 (e-mail: drlokeshsaini@gmail.com).

within normal limits. Biotinidase was found to be severely deficient $(0.69 \mathrm{nmol} / \mathrm{mL}$; range: $5-9 \mathrm{nmol} / \mathrm{mL})$.The spasticity and gait normalized after three months of biotin therapy $(20 \mathrm{mg} /$ day $)$

The clinical and radiological presentations of late-onset biotinidase deficiency are variable. MRI abnormalities are predominantly seen in white matter tracts involving septum pellucidum, corpus callosum, fornix, thalamus, brainstem, periaqueductal gray matter, optic tracts, ${ }^{1}$ hippocampus, and cerebellar white matter. ${ }^{2}$ Diffusion restriction in white matter

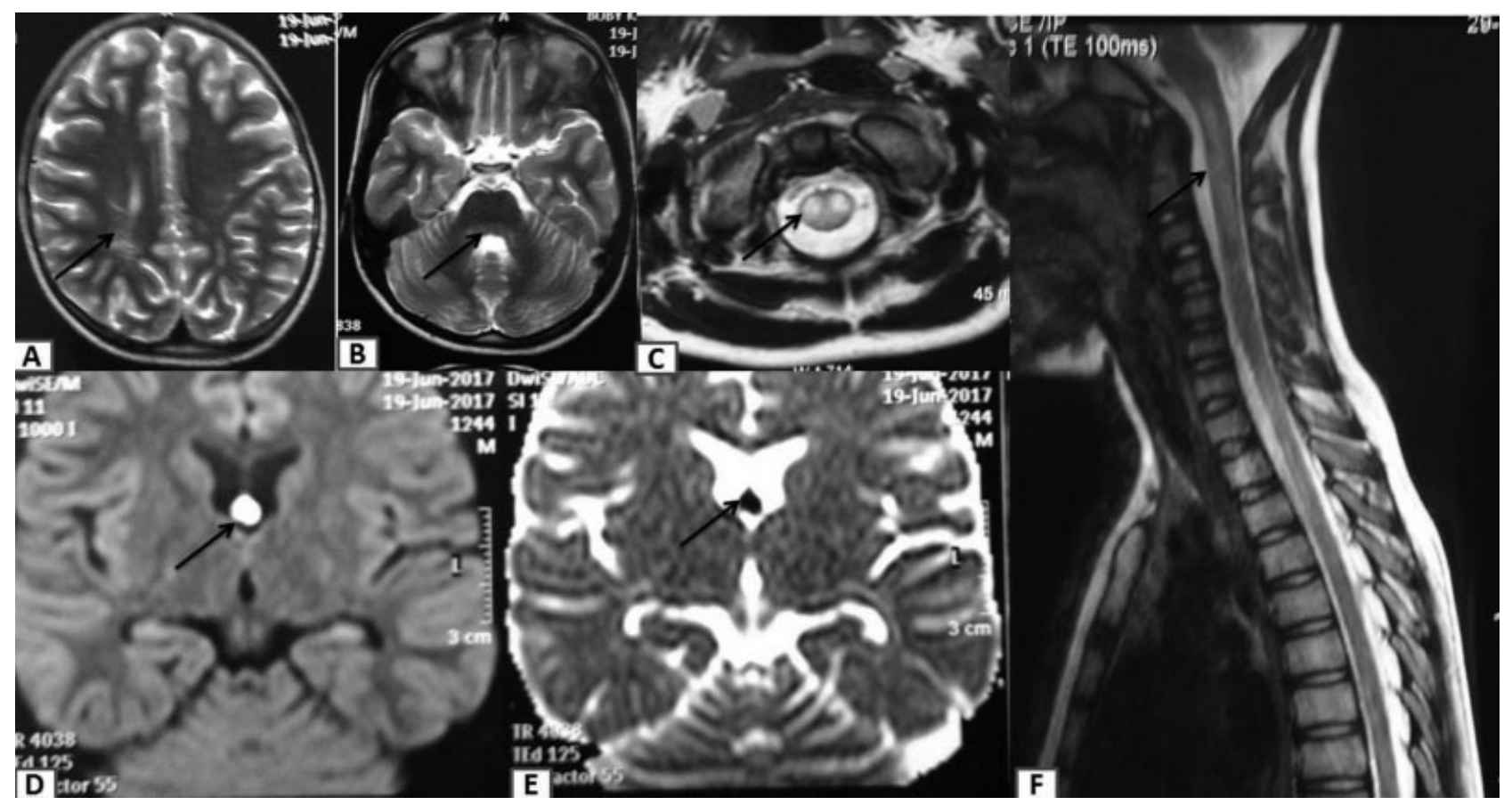

Fig. 1 (A) Magnetic Resonance Imaging showing T2 hyperintensities in periventricular white matter, (B) dorsal pons, (C) In Axial-T2 weighted MRI image of cervical cord, this hyperintensity was confined to anterior part mimicking "Owl's eye”. (D, E) Diffusion restriction was seen in fornix. (F) MRI T2-weighted film, sagittal view of spine, showing longitudnal central T2 hyperintensity from medulla to T1.

received

August 27, 2018

accepted after revision

October 7, 2018

published online

November 19, 2018 (c) 2019 Georg Thieme Verlag KC Stuttgart · New York
DOI https://doi.org/

10.1055/s-0038-1675627. ISSN 0174-304X. 
tracts has been attributed to vacuolating myelinopathy seen in tissue specimens. ${ }^{3}$ Isolated cervicodorsal spine involvement may occur, though whole cord involvement has also been described. ${ }^{1}$ Spinal involvement may be diffuse ${ }^{1}$ or may have selective tract involvement. ${ }^{4}$ Bhat et al have described a child with myelopathy and selective involvement of anterior, lateral, and posterior columns. ${ }^{4}$ Honavar et al have described selective involvement of anterior and posterior column on neuropathology. ${ }^{2}$ The spinal involvement of the index child with selective tract involvement has been radiological described as "Owl's eye appearance", which is typically seen in anterior horn cell myelitis but also described with respect to spinal cord infarction, radiation myelopathy, and fibrocartilaginous emboli. ${ }^{5}$

To conclude, biotinidase deficiency is worth ruling out in all the cases of unexplained spastic quadriparesis or paraparesis as treatment is rewarding.

Authors' Contribution

S.R.D. prepared the initial draft of manuscript and reviewed the literature

N.S. handled patient management, literature review, and editing the initial draft of manuscript

A.K. edited the radiological data, literature review, and edited the initial draft of manuscript

S.V.A. handled analysis of biochemical data, literature review, and editing the initial draft of manuscript L.S. performed critical review of the manuscript and literature review, edited the final version of manuscript and acts as guarantor
Conflict of Interest

The authors have no conflicts of interest to disclose with regard to this article.

Funding

None.

\section{Ethical Approval}

An informed consent form was signed by the parents of the patient to approve the use of patient information or material for scientific purposes. The patient identity has not been disclosed anywhere in the manuscript and doesn't contain any identifiable images.

\section{References}

1 Raha S, Udani V. Biotinidase deficiency presenting as recurrent myelopathy in a 7-year-old boy and a review of the literature. Pediatr Neurol 2011;45(04):261-264

2 Honavar M, Janota I, Neville BG, Chalmers RA. Neuropathology of biotinidase deficiency. Acta Neuropathol 1992;84(04):461-464

3 Van der Knaap MS, Valk J. Multiple carboxylase deficiency. In: Magnetic Resonance of Myelination and Myelin Disorders. 3rd ed. Berlin: Springer-Verlag; 2005:248-251

4 Bhat MD, Bindu PS, Christopher R, Prasad C, Verma A. Novel imaging findings in two cases of biotinidase deficiency-a treatable metabolic disorder. Metab Brain Dis 2015;30(05): 1291-1294

5 Ghosh PS, Mitra S. Owl's eye in spinal magnetic resonance imaging. Arch Neurol 2012;69(03):407-408 